\title{
Influence of Chemical Bonding on the Variability of Diamond-Like Carbon Nanoscale Adhesion: An In-Situ TEM/Nanoindentation and Molecular Dynamics Study
}

Robert W. Carpick ${ }^{1}$, Rodrigo A. Bernal ${ }^{1}$, Polun Chen $^{2}$, J. David Schall ${ }^{3}$, Judith A. Harrison ${ }^{4}$ and YeauRen Jeng ${ }^{2}$

1. Department of Mechanical Engineering and Applied Mechanics, University of Pennsylvania, Philadelphia, PA.

2. Department of Mechanical Engineering, National Chung Cheng University, Chia-Yi, Taiwan.

3. Mechanical Engineering Department, Oakland University, Rochester, MI.

4. Department of Chemistry, United States Naval Academy, Annapolis, MD.

Diamond-like-carbon (DLC) is a versatile and industrially-relevant material for a wide range of tribological applications such as computer hard disk, automotive, machine-tool, and aerospace coatings $[1,2]$. Here, we performed in-situ transmission electron microscopy (TEM) nanoindentation experiments (Figure 1,2) and molecular dynamics (MD) simulations (Figure 3) of nanoscale single asperity contacts composed of tetrahedral amorphous carbon (ta-C, a type of DLC with high strength) in contact with single crystal diamond. Our goal was to understand the factors controlling adhesion [3]. Visualization of the contacts in the TEM-nanoindentation experiments enabled us to correlate the asperity's geometry with the contact and adhesion measurements. The MD simulations enabled atomicscale adhesion mechanisms to be determined and compared with the TEM-nanoindentation observations. Pull-in forces from the experiments show significantly less scatter than the pull-off forces. The magnitude of the pull-in forces is found to be consistent with the adhesion expected from van der Waals (VDW) interactions. This allowed us to estimate the ta-C/diamond Hamaker constant (Figure 2). MD simulations with the AIREBO potential confirmed that including VDW interactions produces less scatter in adhesive forces in approach than in separation, in agreement with the experiments. MD simulations with the REBO $+\mathrm{S}$ potential, which is optimized for modeling bond breaking events, show that the large scatter in the pull-off forces observed experimentally arises from the complex nature of covalent bonding between substrate and tip. The behavior is influenced by the local energy landscape, the hydrogen coverage, and the number of repeated contact events. The scatter in pull-off force also tends to decrease with increasing roughness. From this, we conclude that covalent bonding can play a strong role in influencing the adhesion behavior, producing stochastic variations that must be considered when one seeks to predict adhesive forces for this system.

References:

[1] A.R. Konicek et al, Phys Rev B 85 (2012), p. 155448.

[2] J. Fontaine, C. Donnet, and A. Erdemir in "Tribology of Diamond-Like Carbon Films: Fundamentals and Applications", ed. C. Donnet and A. Erdemir (Springer, New York).

[3] R.A. Bernal et al, Carbon 128 (2018), p. 267. 

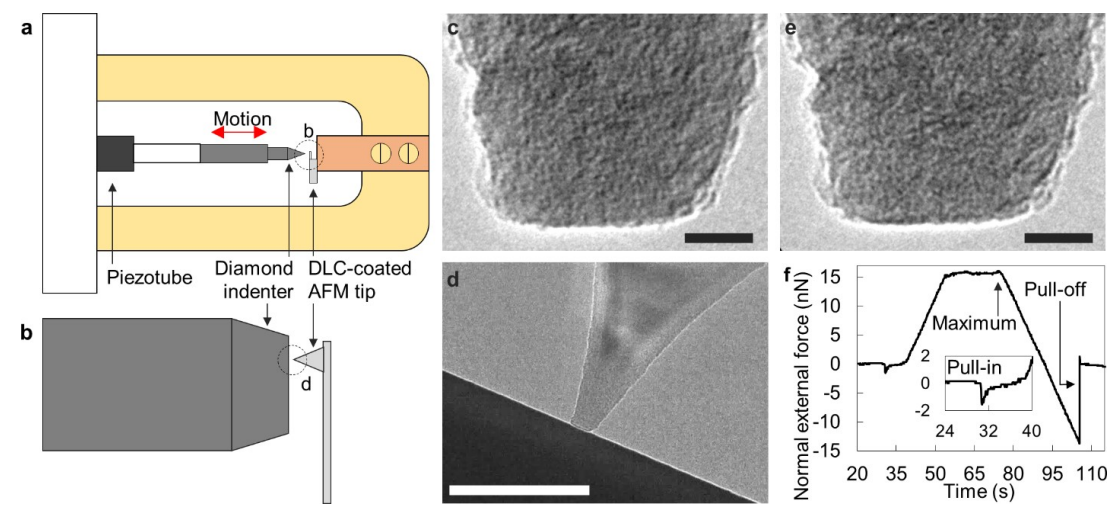

Figure 1. a. Schematic representation of the experimental setup using the Hysitron Picoindenter. The dashed circle is detailed in $\mathbf{b}$, where a schematic close-up of the indenter and AFM cantilever is shown. c. Example of a high-resolution image taken before a contact (scale bar $10 \mathrm{~nm}$ ). d. Frame of a video during contact (scale bar $200 \mathrm{~nm}$ ). e. Example of a high-resolution image taken after a contact (scale bar $10 \mathrm{~nm}$ ). f. Example of a time versus force curve extracted from the TEM videos, showing pull-in force, pull-off force and maximum external force. A close-up of the pull-in event is shown in the inset. $\mathbf{d}$ and $\mathbf{f}$ were obtained from the same contact event.
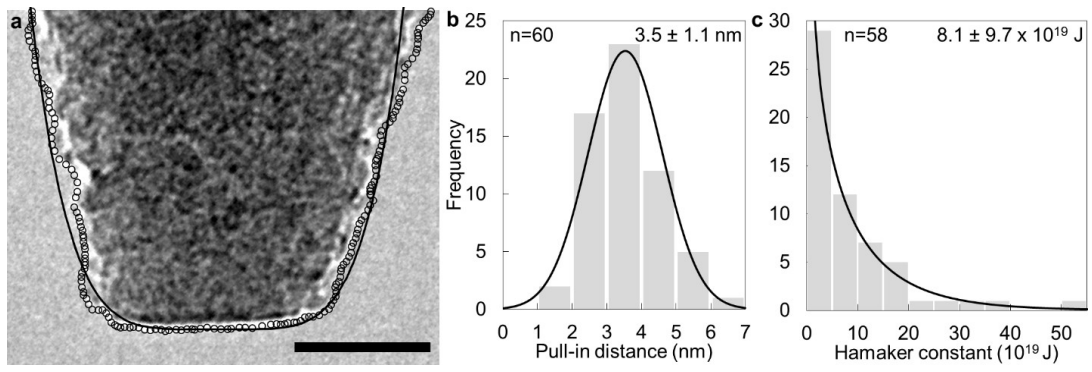

Figure 2. a. High-resolution image of a tip, with overlapped points (o), picked manually, and their fit to a power law function (solid line). Scale bar: $20 \mathrm{~nm}$. b. The pull-in distances exhibit a normal distribution. c. Distribution of the Hamaker constant, fit to a Gamma distribution.
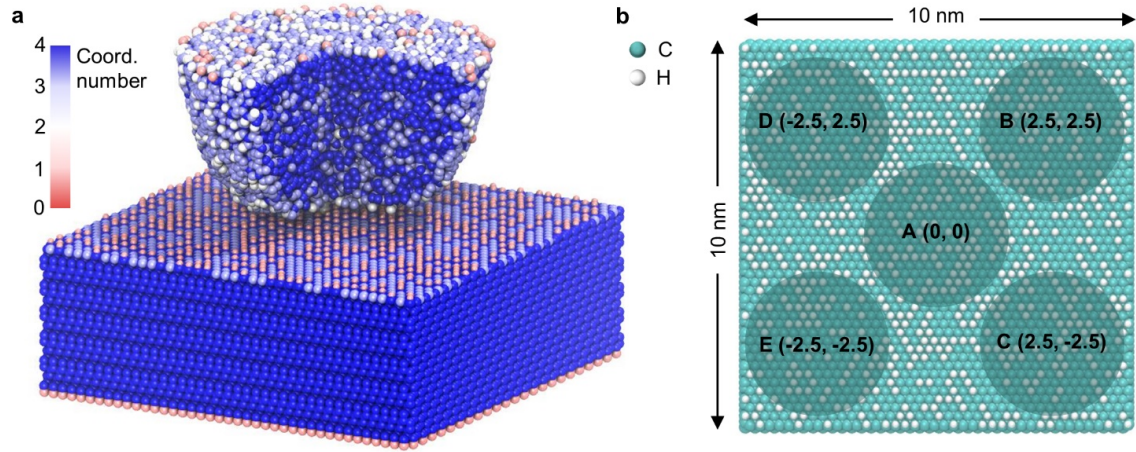

Figure 3. a. Perspective view of the MD simulation setup, with a cross section of the tip to see its internal structure. Small pink spheres represent hydrogen. Carbon atoms are colored by their coordination number. b. Top view of the H-terminated diamond (111) surface. Letters A-E represent different contact points of the tip. 